\title{
Grundtexte zur Freiburger Tradition der Ordnungsökonomik
}

\author{
Hrsg. v. Nils Goldschmidt u. Michael Wohlgemuth
}

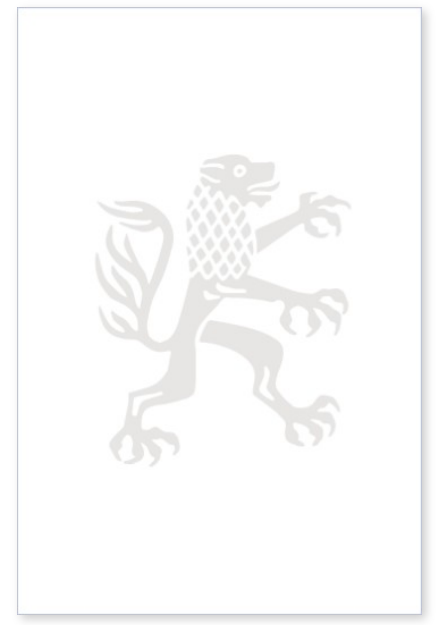

2008. X, 780 Seiten. UOrd 50

ISBN 978-3-16-151476-0

DOI 10.1628/978-3-16-151476-0

eBook PDF 59,00€

ISBN 978-3-16-148297-7

fadengeheftete Broschur 59,00€
Dieser Band bietet anhand von Originaltexten eine Einführung in die Freiburger Tradition der Ordnungsökonomik. Die ausgewählten Texte geben die zentralen Inhalte und Positionen dieser Tradition wieder. Jeder einzelne Text wird ausführlich eingeleitet und kommentiert. Der Band ist in drei Hauptteile gegliedert. Im ersten Teil werden die Ansätze der Vertreter der Freiburger Forschungs- und Lehrgemeinschaft der 1930er und 1940er Jahre dargestellt. Teil zwei widmet sich verwandten Denkansätzen im Umfeld der Freiburger Schule. Im dritten Teil wird die »Freiburger Lehrstuhltradition « präsentiert, also zentrale Texte derjenigen Freiburger Universitätslehrer, die sich in je eigener Akzentuierung mit Fragen von wirtschaftlicher Ordnung und individueller Freiheit beschäftigt haben.

\section{Inhaltsübersicht}

\section{Mit Originaltexten von:}

Franz Böhm, Constantin von Dietze, Ludwig Erhard, Walter Eucken, Edith Eucken-Erdsiek, Hans Gestrich, Hans GroßmannDoerth, Friedrich A. von Hayek, K. Paul Hensel, Joseph Höffner, Erich Hoppmann, Adolf Lampe, Hans Otto Lenel, Elisabeth Liefmann-Keil, Friedrich A. Lutz, Karl Friedrich Maier, Fritz W. Meyer, Leonhard Miksch, Alfred Müller-Armack, Bernhard Pfister, Erich Preiser, Wilhelm Röpke, Alexander Rüstow, Otto Schlecht, Manfred E. Streit, Viktor Vanberg, Erich Welter

Mit Einführungen von:

Detlef J. Blesgen, Gerold Blümle, Patricia Commun, Nils Goldschmidt, Harald Hagemann, Alexander Hollerbach, Karen I. Horn, Wendula Gräfin v. Klinckowstroem, Heinz Lampert, Wernhard Möschel, Werner Mussler, Ursula Nothelle-Wildfeuer, Walter Oswalt, Helge Peukert, Alfred Schüller, Joachim Starbatty, Viktor Vanberg, Reinhold Veit, Christian Watrin, Hans Willgerodt, Michael Wohlgemuth

Nils Goldschmidt ist Professor für Kontextuale Ökonomik und ökonomische Bildung an der Universität Siegen.

Michael Wohlgemuth Geboren 1965; Studium der Volkswirtschaftslehre; 1999 Promotion; 2007 Habilitation; u.a. apl. Professor an der Universität Witten / Herdecke und Forschungsbeauftragter der Stiftung für Staatsrecht und Ordnungspolitik, Liechtenstein.

Jetzt bestellen:

https://mohrsiebeck.com/buch/grundtexte-zur-freiburger-tradition-der-ordnungsoekonomik-9783161514760?no_cache=1 order@mohrsiebeck.com

Telefon: $+49(0) 7071-923-17$

Telefax: +49 (0)7071-51104 